\title{
Sensitivity and Specificity of Upper Extremity Movements Decoded from Electrocorticogram
}

\author{
An H. Do ${ }^{1}$, Po T. Wang ${ }^{2}$, Christine E. King ${ }^{2}$, Andrew Schombs ${ }^{2}$, Jack J. Lin ${ }^{1}$, Mona Sazgar ${ }^{1}$, \\ Frank P.K. Hsu ${ }^{3}$, Susan J. Shaw ${ }^{4,5}$, David E. Millett ${ }^{4,5}$, Charles Y. Liu ${ }^{6,7}$, Agnieszka A. Szymanska ${ }^{2}$, \\ Luis A. Chui ${ }^{1}$, and Zoran Nenadic ${ }^{2,8}$
}

\begin{abstract}
Electrocorticogram (ECoG)-based brain computer interfaces (BCI) can potentially be used for control of arm prostheses. Restoring independent function to BCI users with such a system will likely require control of many degrees-offreedom (DOF). However, our ability to decode many-DOF arm movements from ECoG signals has not been thoroughly tested. To this end, we conducted a comprehensive study of the ECoG signals underlying 6 elementary upper extremity movements. Two subjects undergoing ECoG electrode grid implantation for epilepsy surgery evaluation participated in the study. For each task, their data were analyzed to design a decoding model to classify ECoG as idling or movement. The decoding models were found to be highly sensitive in detecting movement, but not specific in distinguishing between different movement types. Since sensitivity and specificity must be traded-off, these results imply that conventional ECoG grids may not provide sufficient resolution for decoding many-DOF upper extremity movements.
\end{abstract}

\section{INTRODUCTION AND BACKGROUND}

Electrocorticogram (ECoG) has been increasingly used as a signal acquisition modality for brain-computer interface (BCI) applications. Unlike action and local field potentials that rely on intracortical implantation of microelectrodes, ECoG can be acquired using less invasive surgical procedures. Therefore, this signal acquisition modality may have better long-term stability properties.

The majority of ECoG-based BCI studies have focused on decoding the kinematic parameters of upper extremity movements. Examples include decoding and resolving the movement of individual fingers [1], [2], [3], the onset and direction of reaching movements [4], [5], as well as elbow and hand movements [5], [6]. While reaching, grasping and finger movements are important components of nearly all goal-oriented upper extremity tasks, these movements alone are insufficient to regain the upper extremity functions

Work supported by the National Science Foundation (Award \#1134575).

${ }^{1}$ Dept. of Neurology, University of California, Irvine (UCI), Irvine, CA, USA \{and, jjlin, msazgar, lchui\}@uci.edu

${ }^{2}$ Dept. of Biomedical Engineering, UCI, Irvine, CA, USA \{ptwang, kingce, aschombs, aszymans\}@uci.edu

${ }^{3}$ Dept. of Neurosurgery, UCI, Irvine, CA, USA fpkhsu@uci.edu

${ }^{4}$ Dept. of Neurology, Rancho Los Amigos National Rehabilitation Center (RLANRC), Downey, CA, USA

${ }^{5}$ Dept. of Neurology, University of Southern California (USC), Los Angeles, CA, USA $\{$ millett, shaws $\}$ eusc.edu

${ }^{6}$ Dept. of Neurosurgery, RLANRC, Downey, CA, USA

${ }^{7}$ Dept. of Neurosurgery, USC, Los Angeles, CA, USA cliulusc.edu

${ }^{8}$ Dept. of Electrical Engineering and Computer Science, UCI, Irvine, CA, USA znenadic@uci.edu necessary for restoring independence in potential BCI arm prosthesis users. Based on the Functional Independence Measure $^{\mathrm{TM}}$ tool [7], tasks required for independent living, such as dressing, toileting, and transferring, require more complex upper extremity movements. Consequently, the ability to decode six-degree-of-freedom (DOF) movements from ECoG signals may be required to achieve arm prosthesis control capable of restoring independence to those with upper extremity paralysis. However, a comprehensive study of elementary arm movements and their correlation with ECoG signals has yet to be performed.

This study examines the representation of six elementary finger, hand, and arm movements in ECoG signals, and whether these movements can be distinguished from one another. Conceptually, there are two approaches to this problem. In the first approach, a binary classifier may be designed to detect the absence/presence of movement, followed by a multi-class decoder to decide which of the several possible movements was performed. However, this approach renders the movements of individual joints mutually exclusive. The alternative approach used in this study is to design a binary classifier to detect the absence/presence of movement at each joint. An example of detecting wrist movements in real time to drive the "wrist" of a robot arm can be found at: http://youtu.be/DOD3Zyodzby. As seen in the video, the wrist movements can be detected accurately (i.e. high sensitivity), although the model was not very specific, as it would detect movements of other joints. For example, the task in the above video could be accomplished by elbow instead of wrist movements.

Motivated by these observations, this study seeks to formally test the sensitivity and specificity of models for six elementary upper extremity movements. Accurate detection of movement of a particular joint (i.e. sensitivity) is a prerequisite for a reliable detection of the onset of movement. Conversely, the ability to distinguish movements from one another (i.e. specificity) is important for accurate control of multi-joint upper extremity prostheses.

\section{Methods}

\section{A. Overview}

Subjects undergoing subdural ECoG electrode implantation over the primary motor cortex (M1) performed a series of six elementary arm movements while their ECoG signals were recorded. Offline analysis of data corresponding to 
each movement type generated a decoding model to classify ECoG signals into idling or movement states. The classification accuracies of these models were then estimated using cross-validation. In addition, each model's performance in classifying ECoG data from the other five movements was tested. From these classification results, the sensitivity and specificity of each decoding model were determined.

\section{B. Signal Acquisition and Training Data Collection}

This study was approved by the Institutional Review Boards of UCI and RLANRC. Subjects were recruited from an epilepsy patient population undergoing resection surgery evaluation. They had subdural electrodes implanted over the M1 arm representation area. Up to 64 channels of ECoG data were recorded (sampling rate: $2048 \mathrm{~Hz}$, a common average reference) with a pair of linked Nexus-32 bioamplifiers (Mind Media, Roermond-Herten, The Netherlands).

The subjects performed the following elementary movements on the side contralateral to their ECoG electrode implant: (1) pincer grasp/release (PG); (2) wrist flexion/extension (W); (3) forearm pronation/supination (PS), (4) elbow flexion/extension (E); (5) shoulder forward flexion/extension (SFE); (6) shoulder internal/external rotation (SR). For movements PG and W, the trajectory was measured by a custom-made electrogoniometer [8]. The remaining movement trajectories (PS, E, SFE, SR) were measured using a gyroscope (Wii Motion Plus, Nintendo, Kyoto, Japan). All trajectory signals were acquired using an Arduino microcontroller unit (Smart Projects, Turin, Italy). ECoG data were synchronized with the trajectory signals using a common pulse train sent to both acquisition systems. For each movement type, the task consisted of performing 4 sets of 25 continuous movement repetitions, with each set intervened by a 20-30 sec rest (idling) period.

\section{Decoding Model Design and Testing}

To determine the sensitivity of movement detection for each task (encompassing both movement and idling), a decoding model (details in [9]) was designed using the corresponding ECoG data. Briefly, ECoG was synchronized with the motion sensor data using the common pulse train. Subsequently, epochs of idling and movement were delineated based on the trajectory measurements and a suitably chosen threshold. ECoG signals were then labeled accordingly, divided into 0.75 -sec-long trials, and the power spectral density (PSD) of each trial was calculated using the Fourier Transform. PSDs were integrated over the $\mu$ (8-13 $\mathrm{Hz}), \beta(13-30 \mathrm{~Hz})$, low $\gamma(30-50 \mathrm{~Hz})$, and high $\gamma(80-$ $160 \mathrm{~Hz}$ ) bands. These bands were selected because of their involvement in upper extremity movements [10], [11]. To equalize disparate power levels, especially between the $\mu$ and high- $\gamma$ bands, the logarithm of integrated PSDs was taken.

Features were extracted using a combination of classwise principal component analysis (CPCA) [12], [13] and approximate information discriminant analysis (AIDA) [14]. More formally, spatio-spectral features were extracted by:

$$
f=\mathbf{T}_{A} \boldsymbol{\Phi}_{C}(\mathbf{d})
$$

where $f \in \mathbb{R}$ is the feature, $\mathbf{d} \in \mathbb{R}^{B \times C}$ are singletrial spatio-spectral ECoG data ( $B$-the number of frequency bands, $C$-the number of channels), $\boldsymbol{\Phi}_{C}: \mathbb{R}^{B \times C} \rightarrow \mathbb{R}^{m}$ is a piecewise linear mapping from the data space into the $m$-dimensional CPCA-subspace, and $\mathbf{T}_{A} \in \mathbb{R}^{m}$ is an AIDA transformation matrix. These techniques are rooted in information theory [15], [16], and their detailed descriptions can be found in [13], [14]. A linear Bayesian classifier:

$$
f^{\star} \in \begin{cases}\mathcal{I}, & \text { if } P\left(\mathcal{I} \mid f^{\star}\right)>P\left(\mathcal{M} \mid f^{\star}\right) \\ \mathcal{M}, & \text { otherwise }\end{cases}
$$

was then designed in the feature domain, where $P\left(\mathcal{I} \mid f^{\star}\right)$ and $P\left(\mathcal{M} \mid f^{\star}\right)$ are the posterior probabilities of "idling" and "movement" classes, respectively, given the observed feature, $f^{\star}$. They were obtained from the Bayes theorem after assuming $p\left(f^{\star} \mid \mathcal{I}\right)$ and $p\left(f^{\star} \mid \mathcal{M}\right)$ are homoscedastic Gaussian probability density functions. The above procedure was performed for each task, resulting in a total of six decoding models.

For each decoding model, 10 runs of stratified 10-fold cross-validation [17] were performed. The output of the classifier (2) was compared to the class identity of the test data, which generated the following confusion matrix:

\begin{tabular}{c|cc} 
Model $\backslash$ Test & $\mathcal{I}_{a}$ & $\mathcal{M}_{a}$ \\
\hline $\mathcal{I}_{a}$ & $P\left(\mathcal{I}_{a} \mid \mathcal{I}_{a}\right)$ & $P\left(\mathcal{I}_{a} \mid \mathcal{M}_{a}\right)$ \\
$\mathcal{M}_{a}$ & $P\left(\mathcal{M}_{a} \mid \mathcal{I}_{a}\right)$ & $P\left(\mathcal{M}_{a} \mid \mathcal{M}_{a}\right)$
\end{tabular}

Sensitivity, formally defined as $P\left(\mathcal{M}_{a} \mid \mathcal{M}_{a}\right)$, represents the probability of decoding model a correctly classifying the occurrence of movement during task $a$. For example, the highly sensitive model for the PG task would classify all epochs of PG movements as movement class. Similarly, $P\left(\mathcal{I}_{a} \mid \mathcal{I}_{a}\right)$ represents the probability of correctly classifying the occurrence of idling during the same task. Note that the vertical sum of the elements in (3) is 1.

To assess the specificity of model $a$, ECoG data corresponding to task $b(b \neq a)$ were classified using model $a$. This procedure generated the following confusion matrix:

\begin{tabular}{c|cc} 
Model $\backslash$ Test & $\mathcal{I}_{b}$ & $\mathcal{M}_{b}$ \\
\hline $\mathcal{I}_{a}$ & $P\left(\mathcal{I}_{a} \mid \mathcal{I}_{b}\right)$ & $P\left(\mathcal{I}_{a} \mid \mathcal{M}_{b}\right)$ \\
$\mathcal{M}_{a}$ & $P\left(\mathcal{M}_{a} \mid \mathcal{I}_{b}\right)$ & $P\left(\mathcal{M}_{a} \mid \mathcal{M}_{b}\right)$
\end{tabular}

We define specificity as $1-P\left(\mathcal{M}_{a} \mid \mathcal{M}_{b}\right)$, where $P\left(\mathcal{M}_{a} \mid \mathcal{M}_{b}\right)$ represents the probability of model a classifying movement $b$ as movement $a-$ an undesirable outcome. For example, $P\left(\mathcal{M}_{\mathrm{PG}} \mid \mathcal{M}_{\mathrm{W}}\right)$ would be high if the PG model frequently classified $\mathrm{W}$ movement as $\mathrm{PG}$ movement, indicating the low specificity of the model. Note that the specificity can also be expressed as $P\left(\mathcal{I}_{a} \mid \mathcal{M}_{b}\right)$, as elements vertically sum to 1 . Also note that this definition deviates from specificity in the classical sense, defined as $P\left(\mathcal{I}_{a} \mid \mathcal{I}_{a}\right)$. Hence, a more appropriate name would be "cross-specificity," although the term specificity will be retained since the contextual distinction is clear. Similarly, $P\left(\mathcal{I}_{a} \mid \mathcal{I}_{b}\right)$ represents the probability of model $a$ classifying idling epochs as idling during task $b$. If idling behaviors during tasks $a$ and $b$ are similar, this probability would be high. 
TABLE I

THE SENSITIVITY AND SPECIFICITY OF MOVEMENT DECODING FOR SUbJECT S1 (ERROR BARS OMITTED tO SAVE SPACE). THE SHADED BLOCKS ON THE DiAgONAL ARE THE PROBAbILITIES DEFINED By (3) FOR EACH MODEL $a=\{\mathrm{PG}, \mathrm{W}, \mathrm{PS}, \mathrm{E}, \mathrm{SR}, \mathrm{SFE}\}$. THE SENSITIVITY IS THE NUMBER IN THE BOTTOM RIGHT CORNER OF EACH BLOCK. THE OFF-DIAGONAL BLOCKS ARE THE PROBABILITIES DEFINED BY (4), WITH THE SPECIFICITY BEING THE NUMBER IN THE TOP RIGHT CORNER. THE MAXIMUM PER ROW IS MARKED BY $\dagger$, AND THE OVERALL MAXIMUM IS MARKED BY $\ddagger$.

\begin{tabular}{|c|ll|ll|ll|ll|ll|ll|}
\hline Model $\backslash$ Test & $\mathcal{I}_{\mathrm{PG}}$ & $\mathcal{M}_{\mathrm{PG}}$ & $\mathcal{I}_{\mathrm{W}}$ & $\mathcal{M}_{\mathrm{W}}$ & $\mathcal{I}_{\mathrm{PS}}$ & $\mathcal{M}_{\mathrm{PS}}$ & $\mathcal{I}_{\mathrm{E}}$ & $\mathcal{M}_{\mathrm{E}}$ & $\mathcal{I}_{\mathrm{SR}}$ & $\mathcal{M}_{\mathrm{SR}}$ & $\mathcal{I}_{\text {SFE }}$ & $\mathcal{M}_{\text {SFE }}$ \\
\hline $\mathcal{I}_{\mathrm{PG}}$ & 0.96 & 0.04 & 0.92 & 0.12 & 0.89 & 0.24 & 0.93 & 0.21 & 0.78 & 0.24 & 0.94 & $0.57^{\ddagger}$ \\
$\mathcal{M}_{\mathrm{PG}}$ & 0.04 & 0.96 & 0.08 & 0.88 & 0.11 & 0.76 & 0.07 & 0.79 & 0.22 & 0.76 & 0.06 & 0.43 \\
\hline $\mathcal{I}_{\mathrm{W}}$ & 0.88 & 0.10 & 0.91 & 0.02 & 0.84 & 0.08 & 0.93 & 0.05 & 0.68 & 0.04 & 0.91 & $0.23^{\dagger}$ \\
$\mathcal{M}_{\mathrm{W}}$ & 0.12 & 0.90 & 0.09 & $0.98^{\ddagger}$ & 0.16 & 0.92 & 0.07 & 0.95 & 0.32 & 0.96 & 0.09 & 0.77 \\
\hline $\mathcal{I}_{\mathrm{PS}}$ & 0.91 & 0.06 & 0.89 & 0.01 & 0.89 & 0.10 & 0.88 & 0.05 & 0.64 & 0.02 & 0.76 & $0.07^{\dagger}$ \\
$\mathcal{M}_{\mathrm{PS}}$ & 0.09 & 0.94 & 0.11 & 0.99 & 0.11 & 0.90 & 0.12 & 0.95 & 0.36 & 0.98 & 0.24 & 0.93 \\
\hline $\mathcal{I}_{\mathrm{E}}$ & 0.80 & 0.04 & 0.89 & 0.01 & 0.75 & 0.05 & 0.89 & 0.02 & 0.66 & 0.02 & 0.89 & $0.07^{\dagger}$ \\
$\mathcal{M}_{\mathrm{E}}$ & 0.20 & 0.96 & 0.11 & 0.99 & 0.25 & 0.95 & 0.11 & $0.98^{\ddagger}$ & 0.34 & 0.98 & 0.11 & 0.93 \\
\hline $\mathcal{I}_{\mathrm{SR}}$ & 0.97 & $0.23^{\dagger}$ & 0.94 & 0.09 & 0.91 & $0.23^{\dagger}$ & 0.93 & 0.08 & 0.85 & 0.07 & 0.88 & $0.23^{\dagger}$ \\
$\mathcal{M}_{\mathrm{SR}}$ & 0.03 & 0.77 & 0.06 & 0.91 & 0.09 & 0.77 & 0.07 & 0.92 & 0.15 & 0.93 & 0.12 & 0.77 \\
\hline $\mathcal{I}_{\text {SFE }}$ & 0.76 & $0.13^{\dagger}$ & 0.88 & 0.03 & 0.71 & 0.08 & 0.88 & 0.00 & 0.57 & 0.02 & 0.94 & 0.06 \\
$\mathcal{M}_{\mathrm{SFE}}$ & 0.24 & 0.87 & 0.12 & 0.97 & 0.29 & 0.92 & 0.12 & 1.00 & 0.43 & 0.98 & 0.06 & 0.94 \\
\hline
\end{tabular}

TABLE II

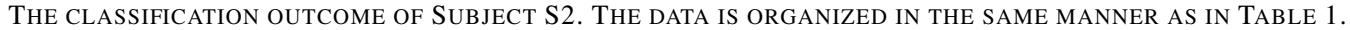

\begin{tabular}{|c|ll|ll|ll|ll|ll|ll|}
\hline Model $\backslash$ Test & $\mathcal{I}_{\text {PG }}$ & $\mathcal{M}_{\text {PG }}$ & $\mathcal{I}_{\mathrm{W}}$ & $\mathcal{M}_{\mathrm{W}}$ & $\mathcal{I}_{\mathrm{PS}}$ & $\mathcal{M}_{\mathrm{PS}}$ & $\mathcal{I}_{\mathrm{E}}$ & $\mathcal{M}_{\mathrm{E}}$ & $\mathcal{I}_{\text {SR }}$ & $\mathcal{M}_{\mathrm{SR}}$ & $\mathcal{I}_{\text {SFE }}$ & $\mathcal{M}_{\text {SFE }}$ \\
\hline $\mathcal{I}_{\mathrm{PG}}$ & 0.90 & 0.01 & 0.94 & 0.01 & 0.88 & 0.02 & 0.86 & 0.31 & 0.78 & 0.00 & 0.80 & $0.84^{\ddagger}$ \\
$\mathcal{M}_{\mathrm{PG}}$ & 0.10 & 0.99 & 0.06 & 0.99 & 0.12 & 0.98 & 0.14 & 0.69 & 0.22 & 1.00 & 0.20 & 0.16 \\
\hline $\mathcal{I}_{\mathrm{W}}$ & 0.73 & 0.00 & 0.94 & 0.00 & 0.83 & 0.01 & 0.78 & 0.23 & 0.71 & 0.00 & 0.64 & $0.75^{\dagger}$ \\
$\mathcal{M}_{\mathrm{W}}$ & 0.27 & 1.00 & 0.06 & $1.00^{\ddagger}$ & 0.17 & 0.99 & 0.22 & 0.77 & 0.29 & 1.00 & 0.36 & 0.25 \\
\hline $\mathcal{I}_{\mathrm{PS}}$ & 0.74 & 0.02 & 0.86 & 0.00 & 0.92 & 0.00 & 0.76 & 0.09 & 0.69 & 0.00 & 0.70 & $0.60^{\dagger}$ \\
$\mathcal{M}_{\mathrm{PS}}$ & 0.26 & 0.98 & 0.14 & 1.00 & 0.08 & $1.00^{\ddagger}$ & 0.24 & 0.91 & 0.31 & 1.00 & 0.30 & 0.40 \\
\hline $\mathcal{I}_{\mathrm{E}}$ & 0.89 & 0.20 & 0.92 & 0.04 & 0.94 & 0.05 & 0.89 & 0.05 & 0.85 & 0.02 & 0.87 & $0.21^{\dagger}$ \\
$\mathcal{M}_{\mathrm{E}}$ & 0.11 & 0.80 & 0.08 & 0.96 & 0.06 & 0.95 & 0.11 & 0.95 & 0.15 & 0.98 & 0.13 & 0.79 \\
\hline $\mathcal{I}_{\mathrm{SR}}$ & 0.88 & 0.03 & 0.93 & 0.00 & 0.95 & 0.03 & 0.89 & 0.25 & 0.87 & 0.01 & 0.82 & $0.70^{\dagger}$ \\
$\mathcal{M}_{\mathrm{SR}}$ & 0.12 & 0.97 & 0.07 & 1.00 & 0.05 & 0.97 & 0.11 & 0.75 & 0.13 & 0.99 & 0.18 & 0.30 \\
\hline $\mathcal{I}_{\mathrm{SFE}}$ & 0.81 & $0.75^{\dagger}$ & 0.67 & 0.23 & 0.73 & 0.21 & 0.78 & 0.04 & 0.67 & 0.07 & 0.93 & 0.02 \\
$\mathcal{M}_{\mathrm{SFE}}$ & 0.19 & 0.25 & 0.33 & 0.77 & 0.27 & 0.79 & 0.22 & 0.96 & 0.33 & 0.93 & 0.07 & 0.98 \\
\hline
\end{tabular}

\section{RESULTS}

Two subjects undergoing subdural electrode implantation for epilepsy surgery evaluation were recruited for this study. Subject S1, a 20-year-old female, was implanted with an $8 \times 8$ grid located on the left anterior frontal temporal area and a $1 \times 6$ posterior frontal strip. To accommodate the 64-channel limit of our data acquisition system (see Section II-B), 6 electrodes on the most anterior column of the $8 \times 8$ grid were excluded. Note that these electrodes were unlikely to carry any motor-related information due to their anterior frontal location. Subject S2, a 28-year-old female, was implanted with a $6 \times 8$ subdural electrode grid in the right frontal parietal area. Each subject was able to complete the 6 tasks described in Section II-B. Their ECoG data were analyzed as described above, and the decoding results are reported in Tables I and II for the two subjects, respectively.

Based on the results from Table I, we conclude that the movements in all six tasks could be detected with high sensitivity for subject $\mathrm{S} 1$, ranging from 0.90 for PS to 0.98 for $\mathrm{W}$ and $\mathrm{E}$ (marked by $\ddagger$ ). The average sensitivity across all models written in the format mean (standard deviation) was $0.95(0.03)$. At the same time, the values of $P\left(\mathcal{I}_{a} \mid \mathcal{I}_{a}\right)$ ranged from $0.85(\mathrm{SR})$ to $0.96(\mathrm{PG})$, averaging $0.91(0.04)$. Similarly, for subject S2 (Table II), the sensitivity ranged from 0.95 (E) to $1.00(\mathrm{~W}, \mathrm{PS})$, with the average of 0.98 (0.02) across all models. Similar to subject $\mathrm{S} 1, P\left(\mathcal{I}_{a} \mid \mathcal{I}_{a}\right)$ ranged from $0.87(\mathrm{SR})$ to $0.94(\mathrm{~W})$ with the average of 0.91 (0.02). Thus, we conclude that the high sensitivity did not compromise the models' ability to correctly detect the idling class, as evidenced by relatively high values of $P\left(\mathcal{I}_{a} \mid \mathcal{I}_{a}\right)$.

Unlike sensitivity, the specificity of individual models was very low. For example, the model for SFE classified all instances of elbow movement as shoulder forward flexion/extension movements (Table I), yielding zero specificity $\left[P\left(\mathcal{I}_{\mathrm{SFE}} \mid \mathcal{M}_{\mathrm{E}}\right)=0.00\right]$. This means that a BCI designed to decode shoulder movement could be operated by elbow movement. On the other hand, the model for PG was somewhat specific with respect to SFE, as it had a specificity of 0.57. On average, the specificity of all models with respect to all movements was $0.11(0.12)$ for subject $\mathrm{S} 1$. Similarly, for subject $\mathrm{S} 2$, the specificity ranged from 0.00 (model/test: PG/SR, W/PG, W/SR, PS/W, PS/SR, SR/W) to 0.84 (model/test: $\mathrm{PG} / \mathrm{SFE}$ ). The average specificity for subject S2 across all models with respect to all movements 
was $0.19(0.26)$. Finally, the probability $P\left(\mathcal{I}_{a} \mid \mathcal{I}_{b}\right)$ averaged 0.84 (0.10) for subject S1, and $0.81(0.09)$ for subject S2, indicating that ECoG signals during idling were similar across all tasks. Note, however, that this is expected, given that idling behavior is similar across tasks, and unlike confusing movements during different tasks, this is considered a positive outcome.

\section{Discussion}

The decoding models generated for each movement type were very sensitive in detecting the presence of movement during all six tasks. Therefore, we conclude that ECoG signals carry a significant amount of information about the six elementary upper extremity movements. This opens the possibility that M1 encoding of complex movements may ultimately be a combination of several elementary movement components. However, this hypothesis will require formal testing. Nevertheless, the high sensitivity reported here may eventually be used to facilitate the future development of ECoG-based BCI-controlled upper extremity prostheses. Note that this may be contingent upon good ECoG grid coverage over M1.

These decoding models had low specificity, i.e. model $a$ had a tendency of confusing movement type $b$ as its own movement. Only in some cases, such as when using the PG model to decode SFE data in subjects S1 and S2, was this confusion somewhat lower (specificity of 0.57 and 0.84 , respectively). The increased specificity for this particular model/test pair is likely due to the somatotopic, anatomical separation of finger from shoulder representation areas on M1. Namely, since the shoulder motor representation area tends to be more medial compared to finger areas, this may lead to a spatial separation between the ECoG features underlying PG and SFE. The SFE movements were the least confused by the other models for both subjects (see the last column of Table I and II). Conversely, the somatotopic proximity of neighboring upper extremity joint representation areas may explain why ECoG features underlying their movements appear to be similar and confuse their respective decoding models. To increase the specificity of these models, the design of the classifier for each movement task may need to involve ECoG data underlying other movements. Given the sensitivity-specificity trade-off, this will likely reduce the sensitivity of these models.

The similarity of idling behaviors across all tasks, as evidenced by the relatively high values of $P\left(\mathcal{I}_{a} \mid \mathcal{I}_{b}\right)$, is not surprising. In instances where this probability was somewhat lower, such as the SFE/SR pair, it can be hypothesized that other factors may have been involved during these idling epochs, such as postural and dynamic stabilization differences, covert movements, or motor planning.

\section{CONCLUSION}

This study demonstrates that the classification of idling and movement in elementary upper extremity movement tasks is highly sensitive, but not specific. While the specificity of these models could be improved, it will likely result in a reduction of sensitivity. These results suggest that conventional ECoG grids do not provide sufficient resolution for decoding 6-DOF upper extremity movements. This problem could be partially mitigated by sacrificing some DOFs, although this may limit the applicability of BCIcontrolled upper extremity prostheses. Alternatively, the use of micro-ECoG electrodes may increase the separability of upper extremity movements, and in turn provide a better decoding resolution.

\section{ACKNOWLEDGMENTS}

The authors acknowledge Angelica Nguyen and Christel Jean for their help in setting up the experiments.

\section{REFERENCES}

[1] J. Kubánek, K.J. Miller, J.G. Ojemann, J.R. Wolpaw, and G. Schalk. Decoding flexion of individual fingers using electrocorticographic signals in humans. J. Neural Eng., 6(6):66001, 2009.

[2] Z. Wang, Q. Ji, K.J. Miller, and G. Schalk. Prior knowledge improves decoding of finger flexion from electrocorticographic signals. Front Neurosci, 5:127, 2011.

[3] N. Liang and L. Bougrain. Decoding finger flexion from band-specific ECoG signals in humans. Front Neurosci, 6:91, 2012

[4] Z. Wang, A. Gunduz, P. Brunner, A.L. Ritaccio, Q. Ji, and G. Schalk. Decoding onset and direction of movements using electrocorticographic (ECoG) signals in humans. Front Neuroeng, 5:15, 2012.

[5] C.M. Chin, M.R. Popovic, A. Thrasher, T. Cameron, A. Lozano, and R. Chen. Identification of arm movements using correlation of electrocorticographic spectral components and kinematic recordings. J Neural Eng, 4(2):146-158, 2007.

[6] T. Yanagisawa, M. Hirata, Y. Saitoh, H. Kishima, K. Matsushita, T. Goto, R. Fukuma, H. Yokoi, Y. Kamitani, and T. Yoshimine. Electrocorticographic control of a prosthetic arm in paralyzed patients. Ann Neurol, 71(3):353-361, 2012.

[7] J. Wright. The FIM ${ }^{\mathrm{TM}}$, The Center for Outcome Measurement in Brain Injury., 2000. http://www.tbims.org/combi/FIM.

[8] P.T. Wang, C.E. King, A.H. Do, and Z. Nenadic. A durable, low-cost electrogoniometer for dynamic measurement of joint trajectories. Med Eng Phys, 33(5):546-552, 2011.

[9] A.H. Do, P.T. Wang, A. Abiri, C.E. King, and Z. Nenadic. Braincomputer interface controlled functional electrical stimulation system for ankle movement. J Neuroeng Rehabil, 8(49), 2011.

[10] K.J. Miller, M. denNijs, P. Shenoy, J.W. Miller, R.P.N. Rao, and J.G. Ojemann. Real-time functional brain mapping using electrocorticography. Neuroimage, 37(2):504-507, 2007.

[11] K.J. Miller, E.C. Leuthardt, G. Schalk, R.P.N. Rao, N.R. Anderson, D.W. Moran, J.W. Miller, and J.G. Ojemann. Spectral changes in cortical surface potentials during motor movement. J Neurosci, 27(9):2424-2432, 2007.

[12] K. Das, S. Osechinskiy, and Z. Nenadic. A classwise PCA-based recognition of neural data for brain-computer interfaces. In Proc. 29th Annual International Conference of the IEEE Engineering in Medicine and Biology Society, pages 6519-6522, 2007.

[13] K. Das and Z. Nenadic. An efficient discriminant-based solution for small sample size problem. Pattern Recogn, 42(5):857-866, 2009.

[14] K. Das and Z. Nenadic. Approximate information discriminant analysis: A computationally simple heteroscedastic feature extraction technique. Pattern Recogn, 41(5):1548-1557, 2008.

[15] Z. Nenadic. Information discriminant analysis: Feature extraction with an information-theoretic objective. IEEE Trans. Pattern Anal. Machine Intell., 29(8):1394-1407, 2007.

[16] Z. Nenadic, D.S. Rizzuto, R.A. Andersen, and J.W. Burdick. Advances in cognitive neural prosthesis: Recognition of neural data with an information-theoretic objective. In G. Dornhege, J.d.R. Millán, T. Hinterberger, D.J. McFarland, and K.-R. Müller, editors, Toward Brain Computer Interfacing, chapter 11, pages 175-190. The MIT Press, 2007

[17] R. Kohavi. A study of cross-validation and bootstrap for accuracy estimation and model selection. In Int. Joint C. Art. Int., pages $1137-$ 1145,1995 